\title{
Colesterolosis y cáncer de la vesícula biliar
}

\author{
IVÁN ROA E. ${ }^{1}$, XABIER DE ARETXABALA U. ${ }^{2}$, \\ GILDA IBACACHE S. ${ }^{1}$, SERGIO MUÑOZ N. ${ }^{3}$
}

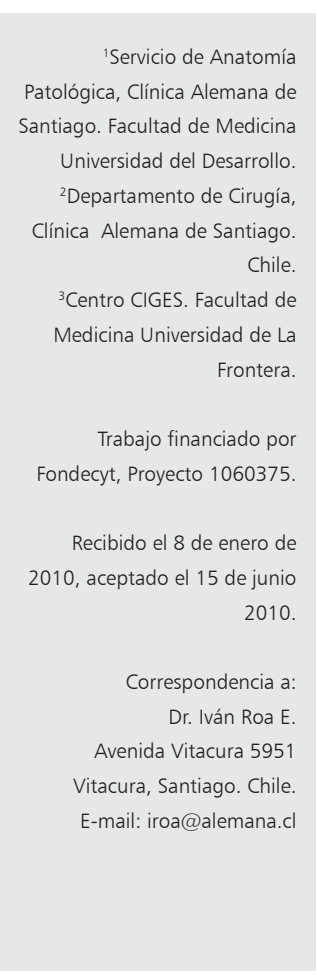

\section{Association between cholesterolosis and gallbladder cancer}

\begin{abstract}
Background: Cholesterolosis is frequently observed in cholecystectomies performed for lithiasis or chronic cholecystitis. Aim: To determine the degree of association between cholesterolosis and gallbladder cancer. Material and Methods: In a prospective study of gallbladder cancer, all gallbladders obtained during cholecystectomies were processed for pathological study, following a special protocol. As part of this study, 23304 surgical samples obtained between 1993 and 2002 were studied, looking for a relationship between cholesterolosis and chronic cholecystitis, adenomas, dysplasia and gallbladder cancer. Results: Seventy nine percent of patients were women. Cholesterolosis was observed in 3,123 cases (13.4\%). Cholesterolosis was more common in women $(14.2 \%)$ than in men $(10.2 \%)(p<0.001)$. In the same period, 29 patients were diagnosed with adenomas (0.12\%), 179 cases with dysplasia not associated with gallbladder cancer $(0.8 \%)$ and 739 gallbladder cancer (3.2\%). The frequency of cholesterolosis was $13.8 \%$ in chronic cholecystitis, $13.7 \%$ in adenomas, $12.1 \%$ in dysplasias and $1.35 \%$ in patients with gallbladder cancer $(p<0.01)$. Of the thirteen cases with gallbladder cancer and cholesterolosis, 10 were early gallbladder carcinomas. Patients with cholesterolosis were 9.2 times less likely to have cancer than those who did not have cholesterolosis. Conclusions: Cholesterolosis has a strong negative association with gallbladder cancer.
\end{abstract}

(Rev Med Chile 2010; 138: 804-808).

Key words: Cholelithiasis; Cholecystectomy; Gallbladder neoplasms.
L a colesterolosis de la vesícula biliar corresponde a la acumulación de lípidos en el citoplasma de histiocitos en el corion de la mucosa. De acuerdo al número y cantidad de estas células, es el aspecto macroscópico que adopta la superficie de la mucosa vesicular. Los acúmulos pueden ser focales llegando en algunos casos a constituir formaciones nodulares o poliposas o pólipos colesterínicos, o bien, adoptar un carácter difuso con patrones reticulares de color amarillo oro.

La colesterolosis es de frecuente observación en el material de colecistectomías realizadas por litiasis ${ }^{1}$, pólipos o por obesidad mórbida, especialmente en mujeres ${ }^{2-5}$. Se ha asociado a sintoma- tología de tipo biliar, sin embargo, la indicación quirúrgica de esta condición es controversial ${ }^{6,7}$.

Respecto de su patogenia, a diferencia de lo que pudiese creerse, no existe gran información, especialmente de tipo morfo-funcional ${ }^{8-11}$. En el estudio histológico de la mucosa con colesterolosis se observa que el epitelio biliar corresponde a epitelio propio y que esta condición tiende a desaparecer en presencia de alteraciones de la mucosa vesicular como las metaplasias.

La incidencia de la colesterolosis es variable dependiendo de las series y de la indicación que haya tenido la colecistectomía, alcanzando hasta $30 \%$ en pacientes con litiasis y hasta $50 \%$ en pacientes obesos mórbidos en algunos países ${ }^{2,4,5,12}$. En 
estos pacientes, otros autores no han demostrado diferencias respecto de vesículas extirpadas por litiasis $^{3}$. Entre los factores predictores de colesterolosis se encuentran: el índice masa corporal, la circunferencia abdominal y la proteína $\mathrm{C}_{\text {reactiva }}{ }^{5}$. También existen estudios que han asociado la presencia de colesterolosis a hipercolesterolemia ${ }^{13,14}$. Otros la han asociado a la saturación de colesterol en la bilis o al contenido de ésteres de colesterol en la mucosa vesicular ${ }^{8,10,11,15-17}$.

Respecto de la relación que pudiese haber entre CVB y colesterolosis, existe muy poca información: encontramos un solo trabajo publicado en japonés en 1993, que describe un caso de CVB asociado a colesterolosis $^{18}$. Sin embargo, de acuerdo a nuestras observaciones iniciales, la colesterolosis es excepcional en vesículas biliares con cáncer, sin que haya evidencia estadísticamente válida para demostrarlo. El objetivo de este trabajo fue determinar el grado de asociación entre la colesterolosis con el CVB en una población de alto riesgo de cáncer vesícular.

\section{Material y Método}

Tipo de estudio: Es parte de un estudio de carácter prospectivo en $\mathrm{CVB}$, iniciado hace dos décadas en el Servicio de Anatomía Patológica del Hospital Temuco, en el cual se desarrolló un protocolo especial de procesamiento para las piezas de colecistectomías con patología tumoral y no tumoral ${ }^{19}$. La metodología y procesamiento se mantuvo uniforme durante todo el período de estudio.

Examen macroscópico: Todas las vesículas biliares fueron abiertas y estiradas en planchas de parafina sólida y fijadas por un período de a lo menos 24 horas. Posteriormente fueron fotografiadas (entre 1987 hasta 1995) o digitalizadas en el período posterior. El examen macroscópico fue enfocado a la búsqueda dirigida de lesiones sospechosas.

Examen microscópico: Se seleccionaron tres muestras representativas de cada uno de los segmentos los que fueron examinados histológicamente para el diagnóstico microscópico rutinario. En presencia de lesiones de preneoplásicas (displasias) o tumorales se procedió al mapeo seriado completo de la pieza quirúrgica.

Casos: Se incluyó el período comprendido entre el 1 de enero de 1993 y el 31 de diciembre de 2002. En dicho período se procesaron 23.304 vesículas biliares. Se determinó la homogeneidad en el diagnóstico de colesterolosis vesicular realizado entre los distintos patólogos, observándose diferencias no significativas en el diagnóstico de colesterolosis en relación al número total de vesículas biliares examinadas por cada uno de ellos.

Análisis estadístico: Se realizó mediante prueba de chi cuadrado para tablas de contingencia, análisis de la varianza para los promedios y determinación de razones riesgo mediante modelos de regresión logística. Se consideró como significativo un $\mathrm{p}<0,05$.

\section{Resultados}

El grupo total estuvo constituido por 23.304 pacientes de los cuales 79 por ciento correspondía a mujeres (18.352) con un promedio de edad de 45,2 años (DE 15,8 años). Los casos restantes correspondieron a hombres (4.952), con un promedio de edad de 53,4 años (DE 16,3 años) ( $\mathrm{p}=$ 0,019 ).

Se observó colesterolosis (Figuras 1 y 2) en 3.123 casos $(13,4 \%)$ del total de colecistectomías, siendo más frecuente entre la $3^{\mathrm{a}}$ y $5^{\mathrm{a}}$ década de la vida, disminuyendo hacia ambos extremos. La máxima frecuencia se observó en la tercera década con $26,2 \%$ de las vesículas biliares examinadas. La colesterolosis fue más frecuente en mujeres $(14,2 \%)$ que en hombres $(10,2 \%)$ $(\mathrm{p}<0,0001)$. Los promedios de edad de las mujeres y hombres con colesterolosis fueron menores que los pacientes sin esta condición $(\mathrm{p}<0,0001$ y 0,008 respectivamente).

Durante el mismo período de tiempo, se diagnosticaron 29 casos con adenomas $(0,12 \%)$, 179 casos con displasia de la mucosa vesicular no asociadas a cáncer $(0,8 \%)$ y 739 pacientes con CVB (3,2\%).

La frecuencia de colesterolosis en relación a los distintos diagnósticos se resumen en la Tabla 1. En pacientes sin patología tumoral (colecistitis crónicas) se observó una frecuencia de colesterolosis de $13,8 \%$. Cifras similares fueron observadas en pacientes con el diagnóstico de adenomas (13,7\%) y en displasias de la mucosa vesicular $(12,3 \%)$. En los pacientes con CVB la colesterolosis estuvo presente en sólo 13 casos (1,35\%). La diferencias 


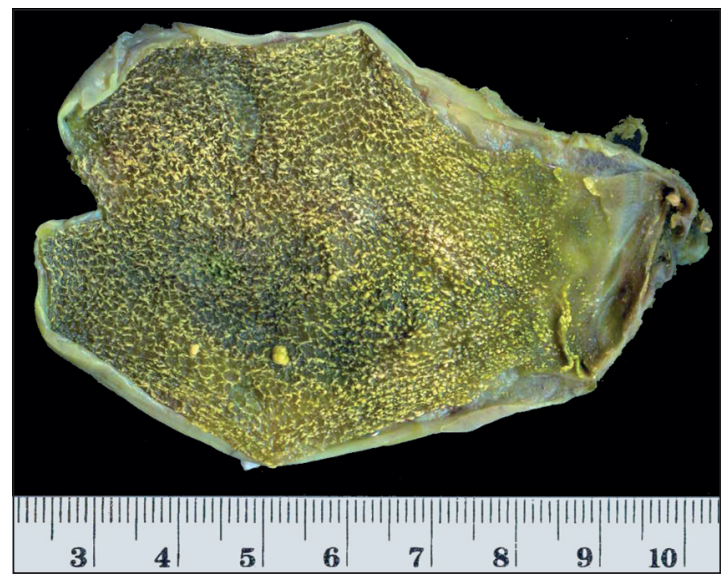

Figura 1. Vesícula biliar extendida y fijada. Se observa colesterolosis difusa de la mucosa en un patrón reticular.

Tabla 1. Frecuencia de colesterolosis de acuerdo al tipo de lesión

\begin{tabular}{|lrrrr|}
\hline \multicolumn{5}{c}{ Colesterolosis } \\
Diagnóstico & \multicolumn{1}{c}{ No } & \multicolumn{1}{c|}{ Sí } & \multicolumn{1}{c|}{ Total } & \% \\
\hline C. crónica & 19.273 & 3.084 & 22.357 & 13,8 \\
Adenoma & 25 & 4 & 29 & 13,8 \\
Displasia & 157 & 22 & 179 & 12,3 \\
Cáncer & 729 & 13 & 739 & 1,35 \\
\hline
\end{tabular}

observadas en la frecuencia de colesterolosis entre los pacientes con CVB respecto de los otros grupos, incluyendo los pacientes con lesiones preneoplásicas y adenomas, fue significativa ( $\mathrm{p}<0,0001)$. En el grupo de 244 pacientes con carcinomas incipientes $(33,1 \%)$ la frecuencia de colesterolosis fue de 4,5\%; en cambio, en los 495 pacientes con cánceres avanzados $(66,9 \%)$ fue de $0,6 \%$ ( $p<0,001)$. De los 13 casos en que se observó concomitantemente cáncer vesicular y colesterolosis, 11 correspondieron a carcinomas incipientes (9 mucosos y 2 musculares) y tres avanzados (subserosos). Las pacientes con carcinomas subserosos y colesterolosis correspondieron a lesiones pequeñas y focalmente infiltrantes. Las tres pacientes tuvieron seguimiento de 54,55 y 77 meses respectivamente, todas las pacientes vivas y sin evidencias de enfermedad tumoral residual al momento del último seguimiento clínico.

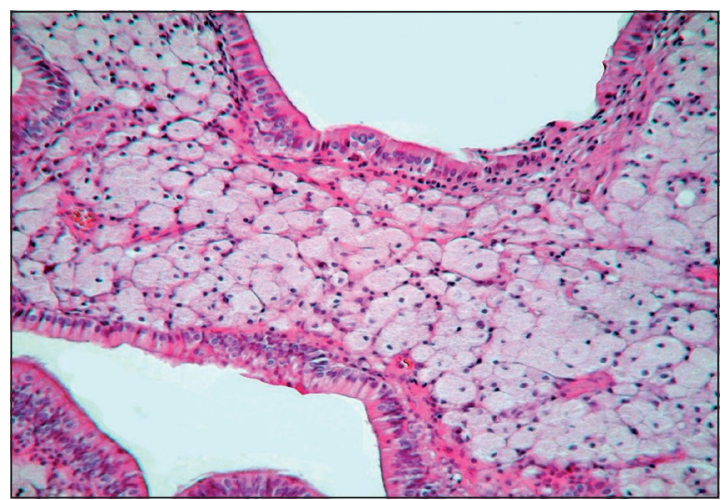

Figura 2. Mucosa vesicular con revestimiento epitelial normotípico, bajo el cual se observa en el corión de la mucosa, gran cantidad de acúmulos de histiocitos espumosos (HE $\mathrm{x}$ 200)

El modelo de regresión logística, ajustado por género y edad mostró una razón de riesgo de 0,887 de tener cáncer y colesterolosis en la misma vesícula biliar (Tabla 2). De esta manera, un paciente con colesterolosis tuvo 9,2 veces menos probabilidad de tener un CVB que aquellos pacientes colecistectomizados de esta serie y que no tenían la presencia de colesterolosis en la mucosa vesicular.

\section{Discusión}

La colesterolosis de la mucosa vesicular es un hallazgo frecuente en el material de colecistectomías por litiasis sintomática. Se observa en alrededor de $15 \%$ de los casos, la mayor parte las veces asociada a cambios mínimos de la mucosa vesicular y escasa inflamación. A medida que aumenta la edad, disminuye su frecuencia. No existe una explicación del por qué de esta asociación negativa, sin embargo, podríamos especular lo siguiente: para que se produzca la colesterolosis, la mucosa debe estar revestida por células con capacidad de concentrar e incorporar el colesterol presente en la bilis a través de un complejo sistema de receptores de membrana ${ }^{10,15}$. La inflamación crónica y la litiasis vesicular producen cambios regenerativos y adaptativos, los que producen el reemplazo del epitelio propio por epitelio metaplásico ${ }^{20,21}$ con la consiguiente pérdida de la capacidad de transportar colesterol. A medida que progresa la intensidad de la metaplasia y la inflamación en la 
Tabla 2. Estimación del riesgo de cáncer y colesterolosis (regresión logística)

\begin{tabular}{|c|c|c|c|}
\hline \multicolumn{4}{|c|}{ Colesterolosis } \\
\hline & Sí & No & Total \\
\hline Cáncer & 13 & 726 & 739 \\
\hline Colecistitis crónica & 3110 & 19455 & 22565 \\
\hline Total & 3123 & 20181 & 23304 \\
\hline \multirow[t]{2}{*}{ Risk } & ,0032051 & ,0361177 & ,0317113 \\
\hline & & Point estimate & [95\% Conf,. Interval] \\
\hline Risk difference & ,0329126 & ,0361621 & 0296631 \\
\hline Risk ratio & 0887412 & ,0476 &, 1654411 \\
\hline Prev. frac. ex. &, 9112588 & 8345589 & ,9524 \\
\hline \multirow[t]{2}{*}{ Prev. frac. Pop } & , 1220017 & & \\
\hline & \multicolumn{2}{|c|}{$\begin{array}{l}\operatorname{chi}^{2}(1)=95,33 \\
\operatorname{Pr}>\operatorname{chi}^{2}=0,0000\end{array}$} & \\
\hline
\end{tabular}

\begin{tabular}{|c|c|c|c|c|}
\hline \multicolumn{5}{|c|}{ Colesterolosis } \\
\hline & Sí & No & Total & Exposed \\
\hline Casos & 13 & 726 & 739 & 0,0135 \\
\hline Controles & 3110 & 19455 & 22565 & 0,1378 \\
\hline Total & 3123 & 20181 & 23304 & 0,1339 \\
\hline \multirow[t]{2}{*}{ Risk } & ,0032051 & 0361177 & ,0317113 & \\
\hline & & Point estimate & [95\% Conf. Interval] & \\
\hline Odds ratio &, 0858111 & ,040932 & , 1589667 & (exact) \\
\hline Risk ratio & & ,8410333 & ,959068 & (exact) \\
\hline Prev. frac. ex. & ,9141889 & & & \\
\hline Prev. frac. Pop & 1259972 & & & \\
\hline & & $\begin{array}{l}\operatorname{chi}^{2}(1)=95,33 \\
\operatorname{Pr}>\operatorname{chi}^{2}=0,0000\end{array}$ & & \\
\hline
\end{tabular}

mucosa vesicular, se produciría la desaparición progresiva de la colesterolosis.

La mucosa vesicular no tumoral adyacente a un CVB, además de presentar inflamación crónica en la totalidad de los casos, presenta displasia, metaplasia, atrofia y excepcionalmente colestero$\operatorname{losis}^{21,22}$. En los aislados casos en que observamos un cáncer de la vesícula biliar asociado a colesterolosis, $80 \%$ de ellos eran de carácter incipiente.

El análisis de regresión logística muestra que la razón de riesgo de tener concomitantemente un cáncer vesicular en presencia de colesterolosis en esta serie, refleja una asociación negativa entre ambas variables. De acuerdo a nuestros resultados, los pacientes cuyas vesículas biliares fueron extirpadas por litiasis sintomática y tenían además colesterolosis en la mucosa vesicular tuvieron 9,2 veces menos probabilidad de tener un cáncer que los pacientes que no presentaban esta condición.

La asociación negativa CVB, también pudiese tener alguna importancia práctica para el cirujano, el cual sólo con la observación macroscópica de la mucosa y la detección de colesterolosis en ella, le permitiría en la gran mayoría los casos prácticamente descartar la presencia de un cáncer vesicular, y si excepcionalmente hubiese uno, en el peor de los escenarios estaría frente a una lesión tumoral incipiente o mínimamente avanzada, de buen pronóstico. 


\section{Referencias}

1. Juvonen T, Niemela O, Makela J, Kairaluoma MI. Characteristics of symptomatic gallbladder disease in patients with either solitary or multiple cholesterol gallstones. Hepatogastroenterology 1994; 41: 263-6.

2. Dittrick GW, Thompson JS, Campos D, Bremers D, Sudan D. Gallbladder pathology in morbid obesity. Obes Surg 2005; 15: 238-42.

3. Csendes A, Burdiles P, Smok G, Csendes P, Burgos A, Recio M. Histologic findings of gallbladder mucosa in 87 patients with morbid obesity without gallstones compared to 87 control subjects. J Gastrointest Surg 2003; 7: 547-51.

4. Fobi M, Lee H, Igwe D, Felahy B, James E, Stanczyk M, et al. Prophylactic cholecystectomy with gastric bypass operation: incidence of gallbladder disease. Obes Surg 2002; 12: 350-3.

5. Liew PL, Wang W, Lee YC, Huang MT, Lin YC, Lee WJ. Gallbladder disease among obese patients in Taiwan. Obes Surg 2007; 17: 383-90.

6. Halldestam I, Enell EL, Kullman E, Borch K. Development of symptoms and complications in individuals with asymptomatic gallstones. Br J Surg 2004; 91: 734-8.

7. Kmiot WA, Perry EP, Donovan IA, Lee MJ, Wolverson RF, Harding LK, et al. Cholesterolosis in patients with chronic acalculous biliary pain. Br J Surg 1994; 81: 112 5.

8. Stromsten A, von Bahr S, Bringman S, Saeki M, Sahlin S, Bjorkhem I, et al. Studies on the mechanism of accumulation of cholesterol in the gallbladder mucosa. Evidence that sterol 27-hydroxylase is not a pathogenetic factor. J Hepatol 2004;40:8-13.

9. Contreras G, Glasinovic JC, González C, Duarte I, Mege RM, Villarroel L. [Association of cholesterolosis and cholelithiasis: pathogenic implications and effects of the natural history of cholelithiasis]. Rev Med Chile 1994; 122: 1158-62.

10. Sahlin S, Stahlberg D, Einarsson K. Cholesterol metabolism in liver and gallbladder mucosa of patients with cholesterolosis. Hepatology 1995; 21: 1269-75.

11. Tilvis RS, Aro J, Strandberg TE, Lempinen M, Miettinen
TA. Lipid composition of bile and gallbladder mucosa in patients with acalculous cholesterolosis. Gastroenterology 1982; 82: 607-15.

12. Csendes A, Smok G, Burdiles P, Díaz JC, Maluenda F, Korn O. Histological findings of gallbladder mucosa in 95 control subjects and 80 patients with asymptomatic gallstones. Dig Dis Sci 1998; 43: 931-4.

13. Khairy GA, Guraya SY, Murshid KR. Cholesterolosis. Incidence, correlation with serum cholesterol level and the role of laparoscopic cholecystectomy. Saudi Med J 2004; 25: 1226-8.

14. Ivanchenkova RA, Sharashkina NV. Heterogeneity of low-density lipoproteins in cholesterolosis of the gallbladder and cholelithiasis. Klin Med 2004; 82: 46-9.

15. Lee J, Choi HS. Reverse cholesterol transport in cultured gallbladder epithelial cells. Korean J Gastroenterol 2004; 43: $145-52$.

16. Satoh H, Koga A. Fine structure of cholesterolosis in the human gallbladder and the mechanism of lipid accumulation. Microsc Res Tech 1997; 39: 14-21.

17. Braghetto I, Antezana C, Hurtado C, Csendes A. Triglyceride and cholesterol content in bile, blood, and gallbladder wall. Am J Surg 1988; 156: 26-8.

18. Fujita N, Noda Y, Kobayashi G, Kimura K, Watanabe H, Suzuki Y, et al. A case of gallbladder cancer associated with cholesterolosis within the cancer region. Nippon Shokakibyo Gakkai Zasshi 1993; 90: 185-9.

19. Roa I, Araya JC, de Aretxabala X, Salinas C, Wistuba I. [Gallbladder pathology in Temuco, IX Region]. Rev Med Chile 1989; 117: 889-94.

20. Albores-Saavedra J, Nadjo M, Henson D, Ziegels-Weissman J, Mones J. Intestinal metaplasia of the gallblader: A morphologic and immunocytochemical study. Hum Pathol 1986; 17: 614-20.

21. Duarte I, Llanos O, Domke H, Harz C, Valdivieso V. Metaplasia and precursor lesions of gallbladder carcinoma. Frequency, distribution, and probability of detection in routine histologic samples. Cancer 1993; 72: 1878-84.

22. Roa I, Araya JC, Wistuba I, Villaseca M, de Aretxabala $\mathrm{X}$, Busel D, et al. [Epithelial lesions associated with gallbladder carcinoma. A methodical study of 32 cases]. Rev Med Chile 1993; 121: 21-9. 